\title{
Equivalent circuit for postcoupler stabilization in a drift tube linac
}

\author{
Francesco Grespan \\ INFN Laboratori Nazionali di Legnaro, Viale dell'università 2, 35020 Legnaro (Padova), Italy
}

(Received 22 September 2011; published 26 January 2012)

\begin{abstract}
Postcouplers (PCs) are devices used in order to reduce the effect of perturbations on the operating mode of a drift tube linac (DTL), using the resonant coupling stabilization method. In this article an equivalent circuit for a DTL equipped with PCs is presented, together with a 3D simulation analysis, which can explain the principle of postcoupler stabilization and define a new tuning strategy for DTL cavities. The PC tuning procedure based on the equivalent circuit and on frequency measurements has been tested and validated with measurements on the Linac4 DTL aluminum model, present at CERN.
\end{abstract}

DOI: 10.1103/PhysRevSTAB.15.010101

PACS numbers: 29.20.Ej

\section{INTRODUCTION}

Drift tube linac (DTL) cavities operate in the $\mathrm{TM}_{010}$ mode. The fields in all the cells have the same phase, so that the overall cell array operates in a 0 mode. The group velocity of a resonating mode is proportional to the slope of the dispersion curve at that point, being $v_{g}=\partial \omega / \partial k_{z}$. Since the group velocity of the $\mathrm{TM}_{010}$ mode is zero, there is no power flowing along the structure and the field distribution is very sensitive to frequency perturbations of the cells, power losses, and beam loading. This problem is particularly serious for long DTLs, where the frequency separation between modes is lower.

A solution has been proposed in the 1960's at Los Alamos [1], applying the resonant coupling method. In this case the resonant coupling elements are simple internal metallic bars, which extend from the outer cylinder towards the drift tube (DT), without touching the latter (Fig. 1). These bars are called postcouplers (PCs).

The postcouplers are located at the points on the outer wall of the cavity that are aligned with the centers of the drift tubes, and oriented at $90^{\circ}$ with respect to the stems, in order to minimize the coupling between posts and stems. Furthermore, the nature of the coupling between adjacent PCs is such that they must be placed on opposite sides of the cavity (Fig. 1).

The system, composed of two chains of coupled resonators, has two bands of frequencies: the TM band and the PC band (Fig. 2).

The confluence mode of the TM passband and the PC passband is the 0 mode. However, since the PC mode field distribution corresponds to a capacitively loaded TE mode, the PC 0 mode cannot be excited in a real tank with conducting end walls, but it is allowed only in an infinite structure (Fig. 3). The PC 0 mode has the electric field from

Published by the American Physical Society under the terms of the Creative Commons Attribution 3.0 License. Further distribution of this work must maintain attribution to the author(s) and the published article's title, journal citation, and DOI. postcouplers to drift tubes all with the same direction and magnitude as it can be simulated using perfect magnetic end walls. In the case of metallic walls, the electric field magnitude is not the same for each postcoupler, therefore this is not a "pure" 0 mode.

Figure 4 shows simulated TM and PC bands of structures in Fig. 3. The plot distinguishes between modes allowed with perfect magnetic boundary conditions and modes allowed with perfect electric boundary conditions. It is interesting to notice in Fig. 4 that the three PC modes simulated with perfect electric boundary conditions have the same frequencies as the three central modes of the PC band simulated with perfect magnetic boundary conditions.

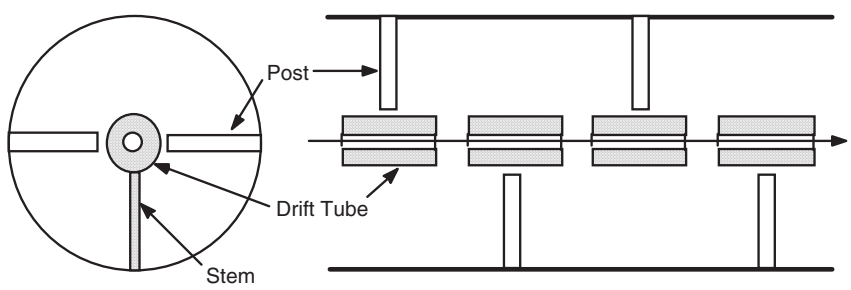

FIG. 1. Postcouplers inside a DTL cavity.

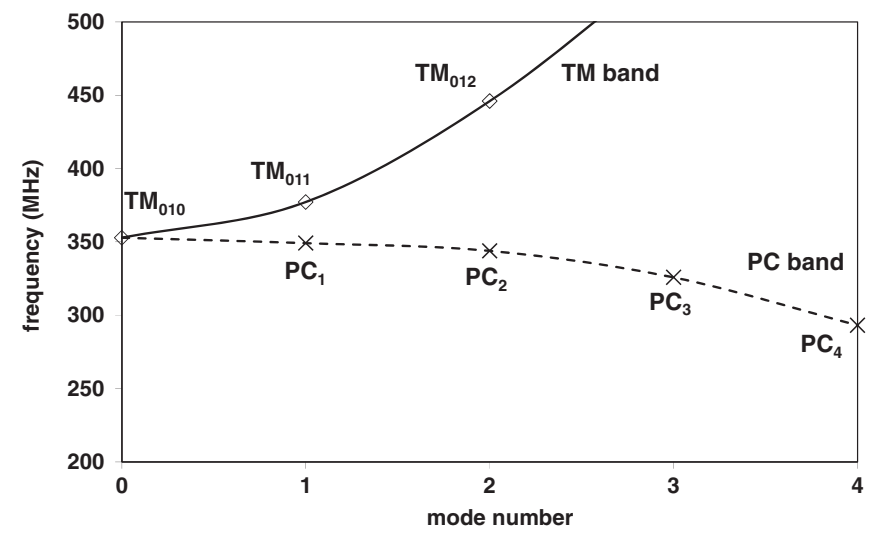

FIG. 2. TM band associated to the accelerating cells and PC band associated to postcoupler resonators. 

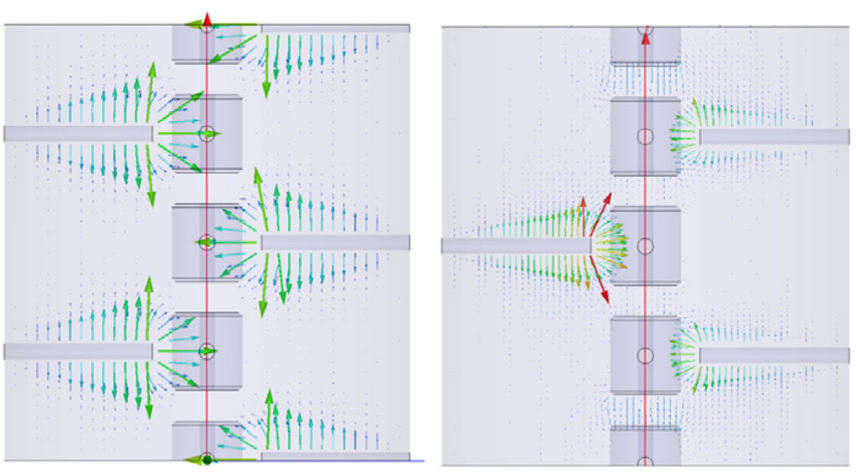

FIG. 3. The PC 0 mode can be simulated only with perfect magnetic end walls (left). With metallic end walls (right) it is not possible to excite a "pure" PC 0 mode.

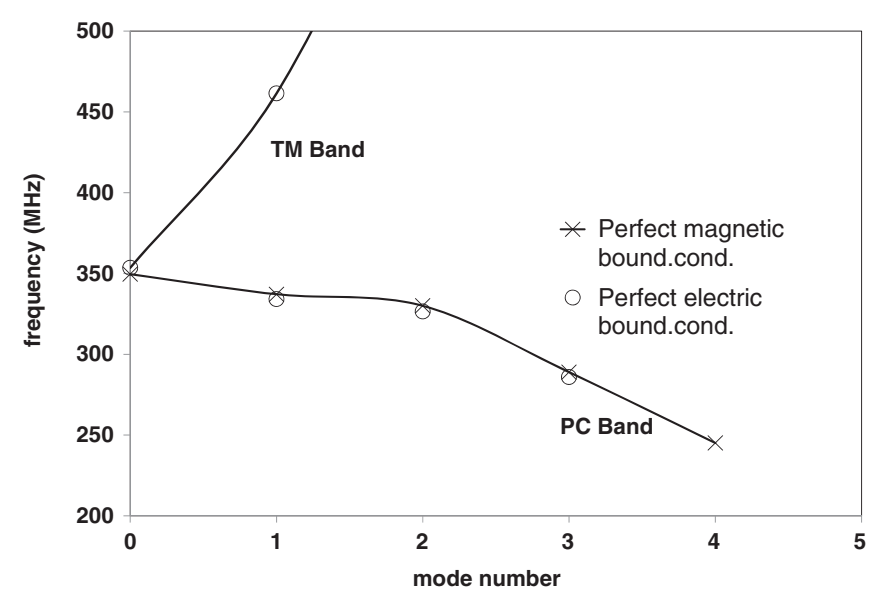

FIG. 4. Dispersion curves simulated with perfect electric boundary conditions and with perfect magnetic boundary conditions in a DTL with postcouplers, as shown in Fig. 3. Only perfect magnetic boundary conditions allow the $\mathrm{PC}_{0}$ mode excitation.

The coupling between postcouplers and DTL accelerating cells is provided by the capacitance from the end of the PCs to the drift tube walls. The coupling is stronger if the gap between the PCs and the drift tubes is small. The tuning of the coupling element resonance to get the confluence point can be done by adjusting the distance between postcouplers, for example, changing the number of postcouplers per drift tube. Once this parameter is determined, it is still possible to tune the postcoupler frequency by changing the gap size between the postcouplers and the drift tubes. It is clear that a variation of the gap changes the capacitive coupling strength as well.

\section{POSTCOUPLER GEOMETRICAL PARAMETERS}

Three-dimensional HFSS [2] simulations have been used in order to obtain values of the $\mathrm{PC}_{1}$ mode frequency as a function of the number of PC per unit length of the tank (Fig. 5). The magnetic and electric field pattern of PC
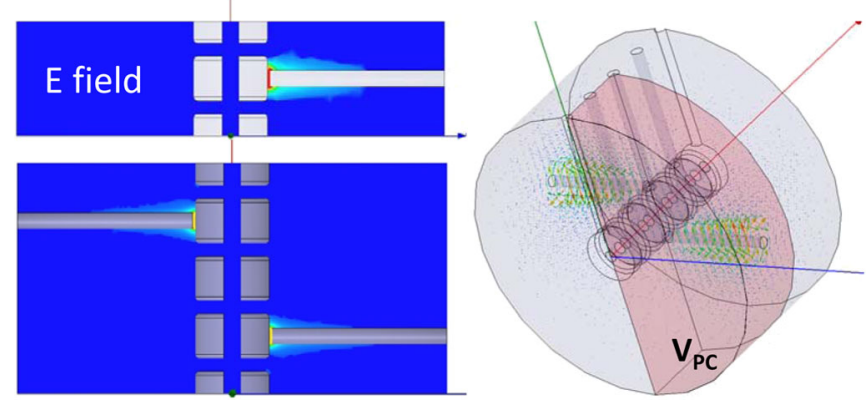

FIG. 5. On the left side, electric fields of two cavity configurations equivalent on number of PCs per unit length is shown. On the right side, the shadowed volume is $V_{\mathrm{PC}}$, used for the definition of the equivalent diameter $D_{\text {eq }}$ of the inductor $L_{p}$.

modes (Fig. 5) suggests a circuital view of PCs: a series inductance capacitance, resonating at the frequency $f_{p}=\frac{1}{2 \pi \sqrt{L_{p} C_{p}}}$.

Taking a very small gap between PC and DT (gap PC-DT $\ll \lambda / 4$ ), the PC mode electric field is concentrated in the gap area, so it is possible to apply the quasistatic approximation [3] and define an electric field potential $V$ in order to calculate values of the capacitance $C_{p}$ associated to the gap PC-DT using the formula $C_{p}=\frac{2 U}{V^{2}}$, where $U$ and $V$ are calculated from the simulation. For a gap PC-DT $=$ $3 \mathrm{~mm}$, the results show a value of $C_{p}$ distributed with respect to an average value of $\bar{C}_{p}=(2.8 \pm 0.2) \mathrm{pF}$, while the $\mathrm{PC}_{1}$ mode frequency increases with the number of $\mathrm{PCs}$ per unit length (Fig. 6). The uncertainty on the $C_{p}$ calculation is due to the mesh on the gap PC-DT, which affects the integral $V=\int_{\text {gap PC-DT }} \vec{E}(s) \cdot d \vec{s}$.

For small variations of the distance between PC and DT, the inductance $L_{p}$ associated to the PC is a function of the distance between PCs. Figure 7 shows the values of $\mathrm{PC}$ inductance $L_{p}$ calculated from simulations as

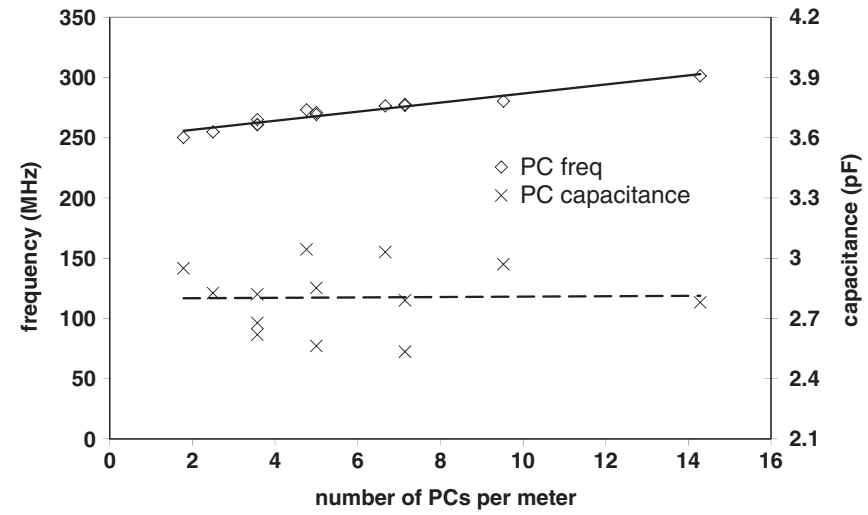

FIG. 6. $\mathrm{PC}_{1}$ mode frequency and PC capacitance $C_{p}$ from 3D simulations of different distributions of PCs inside the tank. Simulation settings are: gap PC-DT $=3 \mathrm{~mm}$, drift tube radius $=$ $45 \mathrm{~mm}$, tank radius $=260 \mathrm{~mm} ; \mathrm{PC}$ radius $=10 \mathrm{~mm}$. 


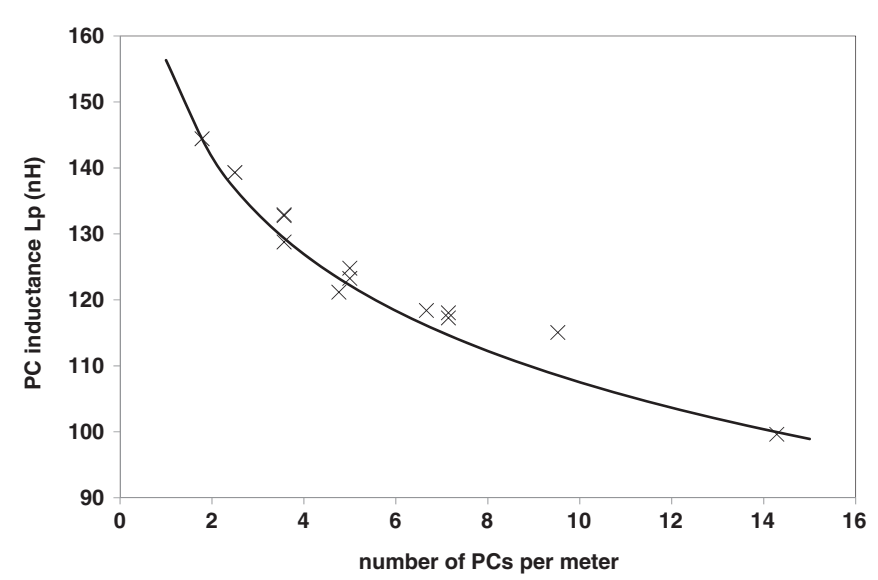

FIG. 7. PC inductance $L_{p}$ as a function of the number of PCs per meter. The solid line is given by the $L_{p}$ formula, crosses are obtained from 3D simulations in quasistatic approximation.

$L_{p}=\frac{1}{\left(2 \pi f_{p}\right)^{2} \bar{C}_{p}}$ compared with the curve obtained by the formula $L_{p}=2 \times 10^{-7} \ln \left(\frac{D_{\mathrm{eq}}}{d_{\mathrm{PC}}}\right) \cdot l_{\mathrm{PC}}$. This formula considers a PC as a coaxial inductor where the PC itself is the inner conductor and the outer conductor has an equivalent diameter of $D_{\text {eq }}=D_{\text {tank }} \sqrt{L_{\text {tank }} /\left(2 \cdot N_{\mathrm{PCs}}^{o} \cdot l_{\mathrm{PC}}\right)}$ (Fig. 5). $D_{\text {eq }}$ is the diameter of a cylinder obtained from the evaluation of the volume $V_{\mathrm{PC}}$ filled up by the magnetic field lines of each PC: $V_{\mathrm{PC}} \equiv \frac{1}{2}\left\{\left[\pi\left(D_{\mathrm{tank}} / 2\right)^{2} \cdot L_{\mathrm{tan} k}\right] / N_{\mathrm{PCs}}^{\circ}\right\}=$ $\pi\left(D_{\text {eq }} / 2\right)^{2} \cdot l_{\mathrm{PC}}$, where $L_{\text {tank }}$ and $D_{\text {tank }}$ are the tank length and diameter, $l_{\mathrm{pc}}$ is the PC length, and $N^{\circ}{ }_{\mathrm{PCs}}$ is the number of PCs inside the tank.

In quasistatic conditions it is possible to define an electric field potential $V$, and then to estimate the $C_{p}$ value starting from electrostatic considerations. The electrostatic definition for the capacitance is $C=\frac{Q}{V}=\frac{\oint_{A} \sigma \cdot d A}{\int_{d} \bar{E} \cdot d \bar{s}}=$ $\frac{\varepsilon_{0} \oint_{A} \bar{E} \cdot d \bar{A}}{\int_{d} \bar{E} \cdot d \bar{s}}$. We want to get a simple empirical formula $C_{p}=$ $\frac{\varepsilon_{0} A_{Q}}{g_{\mathrm{av}}}$, where $A_{Q}$ is the area where the surface charge density $\sigma$ induced by the electric field is distributed, and $g_{\text {av }}$ is the average integral path of the electric field. Figure 8 shows in detail the electric field distribution between PC and DT in a PC mode. The surface charge on the DT is distributed over

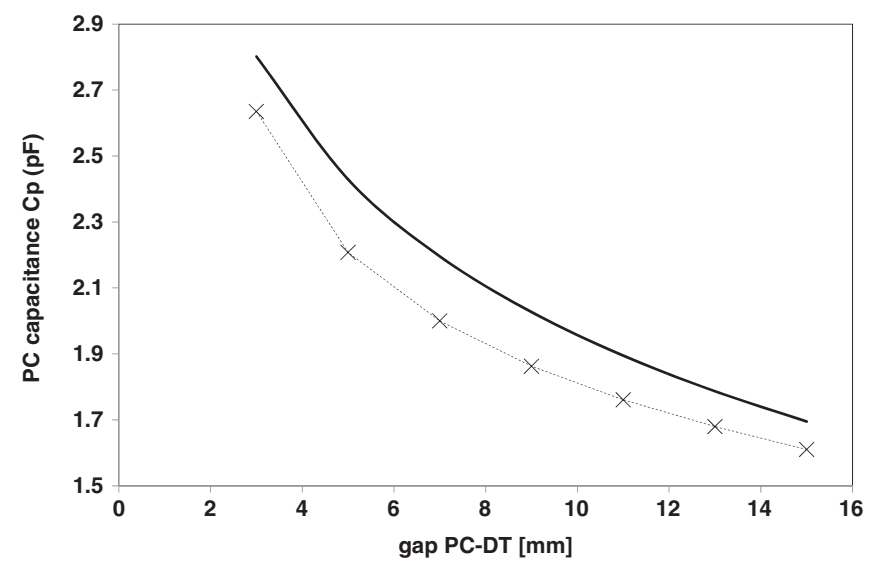

FIG. 9. PC capacitance $C_{p}$ as a function of the gap PC-DT. The solid line is given by the $C_{p}$ formula, crosses are obtained from $3 \mathrm{D}$ simulations in quasistatic approximation.

an ellipsis of area $A_{Q}=\left(\pi \cdot x_{\mathrm{tg}} \cdot s_{\mathrm{tg}}\right)$ and the average distance from the PC is $g_{\mathrm{av}}=\frac{1}{2}\left(d_{\mathrm{DT}}+2 g-y_{\mathrm{tg}}\right)$, where $P_{\mathrm{tg}}=\left(x_{\mathrm{tg}}, y_{\mathrm{tg}}\right)$ is the tangent point to the drift tube cylinder of a line starting from the border tip point of the PC, $s_{\mathrm{tg}}$ is the arc length from $P_{\mathrm{tg}}$ to the top of the drift tube, $d_{\mathrm{DT}}$ is the DT diameter, and $g$ is the gap PC-DT. The capacitance is independent from the length of the DT and the formula is

$$
C_{p}=\varepsilon_{0} \frac{A_{Q}}{g_{\mathrm{av}}}=\varepsilon_{0} \frac{\pi \cdot x_{\mathrm{tg}} \cdot d_{\mathrm{DT}} \arccos \left(2 y_{\mathrm{tg}} / d_{\mathrm{DT}}\right)}{\frac{d_{\mathrm{DT}}}{2}+2 g-y_{\mathrm{tg}}} .
$$

Figure 9 shows the curve for $C_{p}$ given by the formula with the values calculated from simulations as $C_{p}=\frac{2 U}{V^{2}}$. The equation overestimates the capacitance by about $10 \%$ with respect to the simulation results.

The interaction of PCs and stems has also been studied with 3D simulations, in order to properly insert stems in the DTL equivalent circuit.

Stem modes can be distinguished in $\mathrm{rf}$ measurements because of the much lower frequencies with respect to the operating mode and because of the low sensitivity to gap PC-DT displacements. Three-dimensional simulations show that the presence of DT stems weakly affects the field pattern of the PC modes (Fig. 10): the electromagnetic energy is concentrated around the PCs, with a slight

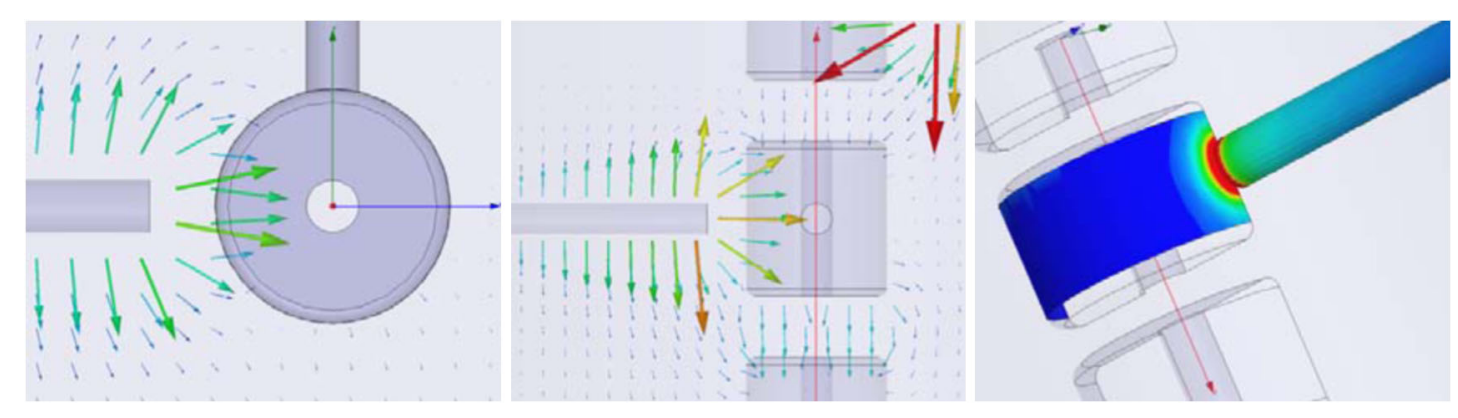

FIG. 8. Electric field pattern between PC and DT in a PC mode. 


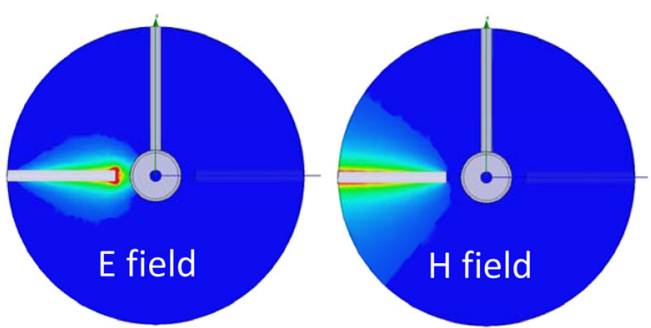

FIG. 10. $\mathrm{E}$ and $\mathrm{H}$ field magnitude of the PC1 mode showing weak influence of DT stems on PC mode pattern: PC field lines do not intersect DT stems.

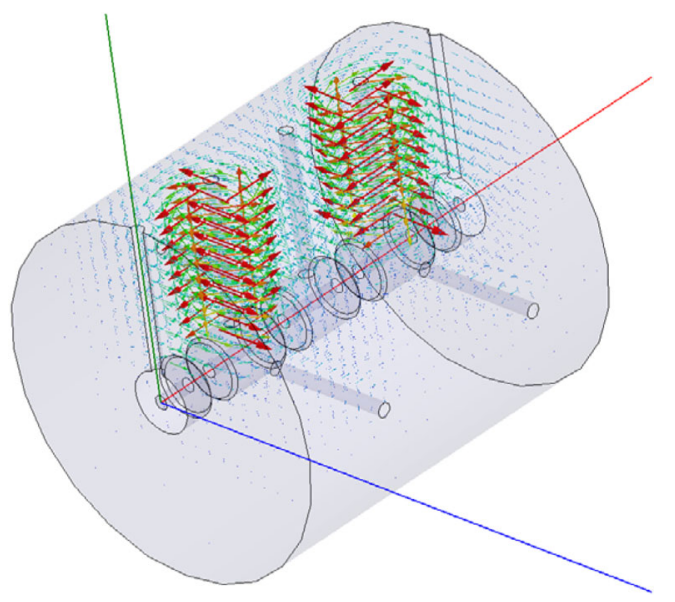

FIG. 11. A 3D view of stem mode magnetic field, showing weak coupling between stems and PCs: stem field lines do not intersect PCs.

deviation of the $\mathrm{H}$ field around the stems, and the change in mode frequency can be estimated using the Slater perturbation theorem. The same behavior can be noticed for the stem modes in relation to the presence of PCs (Fig. 11).

From this we conclude that DT stems and PCs can be considered separately in the equivalent circuit.

\section{EQUIVALENT CIRCUIT}

A complete equivalent circuit for a DTL cell equipped with PCs is shown in Fig. 12. In this circuit $C_{0}$ represents the gap capacitance, $L_{0} / 2$ represents the inductance of half drift tube (DT), and $C_{s}$ is the capacitance of a DT to the tank wall, represented by the ground conductor. Inductors $L_{s}$ represent stems, in parallel with the shunt capacitance $C_{s}$, which represents the electric field from the DT to the tank. $C_{p}$ is the capacitance between PC and DT and $L_{p}$ the inductance of a PC.

There are 3 main resonator chains in a DTL: drift tube resonators, stem resonators, and PC resonators. Measurements on the Linac4 DTL prototype [4] show that the stem dispersion curve is lower than TM and PC dispersion curves (Fig. 13). Since for frequencies close to the operating mode the stem impedance $Y_{\text {stem }}=$ $j \omega C_{s}\left(1-\frac{\omega_{s}^{2}}{\omega^{2}}\right)$ is capacitive and there is no coupling

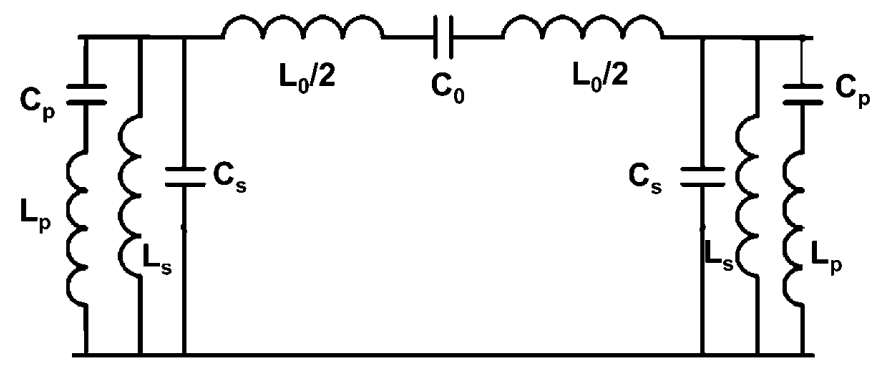

FIG. 12. Equivalent circuit for a DTL including DT, stem, and $\mathrm{PC}$ resonators.

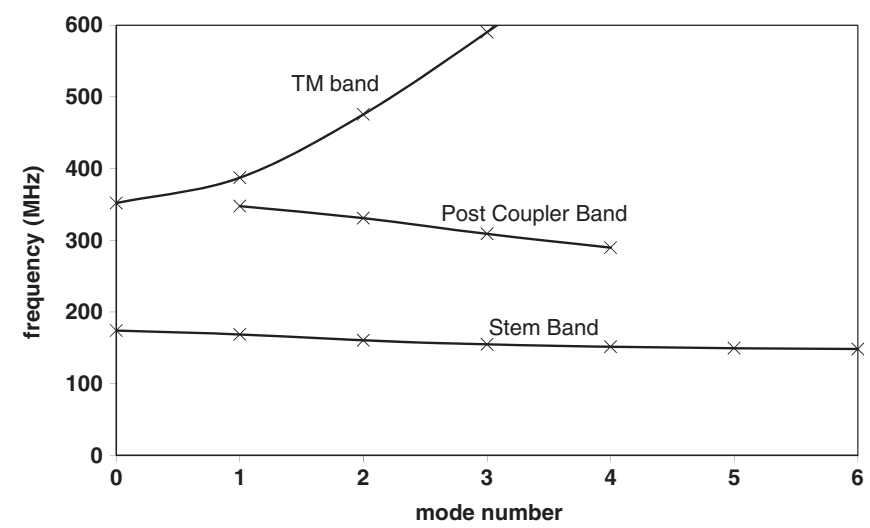

FIG. 13. Frequency bands in the DTL prototype.

between stem and PC, the circuit model is simplified to the circuit in Fig. 14, where the capacitance $C$ is defined $C=C_{s}\left[1-\left(\omega_{s}^{2} / \omega^{2}\right)\right]$.

Solving the mesh equations for the drift tube and the PC currents, we obtain the system

$i p_{n}\left(j \omega L_{p}+\frac{1}{j \omega C_{p}}\right)-\left(i_{n-1}-i_{n}-i p_{n}\right) \frac{1}{j \omega C}=0$

$i_{n}\left(j \omega L_{0}+\frac{1}{j \omega C_{0}}\right)+\left(2 i_{n}-i_{n-1}-i_{n+1}+i p_{n}-i p_{n+1}\right)$

$\times \frac{1}{j \omega C}=0$

$i p_{n+1}\left(j \omega L_{p}+\frac{1}{j \omega C_{p}}\right)-\left(i_{n}-i_{n+1}-i p_{n+1}\right) \frac{1}{j \omega C}=0$.

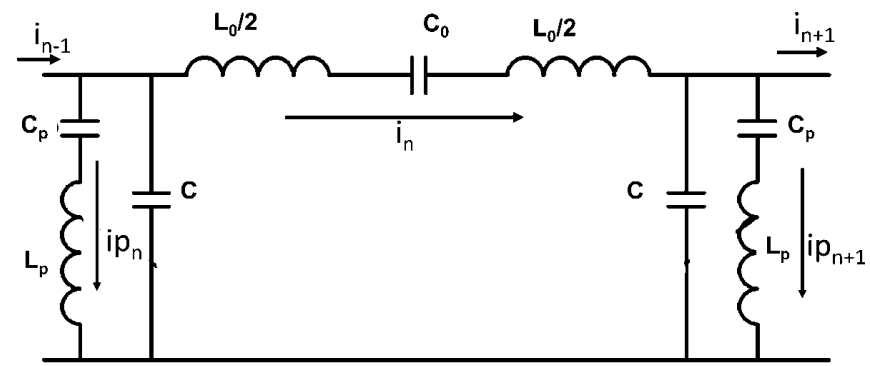

FIG. 14. Equivalent circuit for a DTL equipped with PCs. 
This system is equivalent to the following, with some substitutions and simplifications:

$$
\begin{aligned}
-\frac{i_{n-1}}{C}+i p_{n}\left(\frac{1}{C}+\frac{1}{C_{p}}\right)+\frac{i_{n}}{C} & =\omega^{2} \frac{i p_{n}}{C_{p} \omega_{p}^{2}} \\
-\frac{i_{n-1}}{C}+\frac{i p_{n}}{C}+i_{n} \cdot\left(\frac{2}{C}+\frac{1}{C_{0}}\right)-\frac{i p_{n+1}}{C}-\frac{i_{n+1}}{C} & =\omega^{2} \frac{i_{n}}{C_{0} \omega_{0}^{2}} \\
-\frac{i_{n}}{C}+i p_{n+1}\left(\frac{1}{C}+\frac{1}{C_{p}}\right)+\frac{i_{n+1}}{C} & =\omega^{2} \frac{i p_{n+1}}{C_{p} \omega_{p}^{2}} .
\end{aligned}
$$

This system of equations can be put into a matrix form $\overline{\bar{M}} \cdot \vec{I}=\omega^{2} \vec{I}$, to be solved as an eigenvalue problem. For a cavity of 3 cells +2 PCs the $5 \times 5$ matrix is

$$
\left[\begin{array}{ccccc}
C_{0} \omega_{0}^{2}\left(\frac{1}{C}+\frac{1}{C_{0}}\right) & -\frac{C_{p} \omega_{p}^{2}}{C} & -\frac{C_{0} \omega_{0}^{2}}{C} & 0 & 0 \\
-\frac{C_{0} \omega_{0}^{2}}{C} & C_{p} \omega_{p}^{2}\left(\frac{1}{C}+\frac{1}{C_{p}}\right) & \frac{C_{0} \omega_{0}^{2}}{C} & 0 & 0 \\
-\frac{C_{0} \omega_{0}^{2}}{C} & \frac{C_{p} \omega_{p}^{2}}{C} & C_{0} \omega_{0}^{2}\left(\frac{2}{C}+\frac{1}{C_{0}}\right) & -\frac{C_{p} \omega_{p}^{2}}{C} & -\frac{C_{0} \omega_{0}^{2}}{C} \\
0 & 0 & -\frac{C_{0} \omega_{0}^{2}}{C} & C_{p} \omega_{p}^{2}\left(\frac{1}{C}+\frac{1}{C_{p}}\right) & \frac{C_{0} \omega_{0}^{2}}{C} \\
0 & 0 & -\frac{C_{0} \omega_{0}^{2}}{C} & \frac{C_{p} \omega_{p}^{2}}{C} & C_{0} \omega_{0}^{2}\left(\frac{1}{C}+\frac{1}{C_{0}}\right)
\end{array}\right] \cdot\left[\begin{array}{c}
\frac{i_{0}}{C_{0} \omega_{0}^{2}} \\
\frac{i p_{1}}{C_{P} \omega_{P}^{2}} \\
\frac{i_{1}}{C_{0} \omega_{0}^{2}} \\
\frac{i p_{2}}{C_{P} \omega_{P}^{2}} \\
\frac{i_{2}}{C_{0} \omega_{0}^{2}}
\end{array}\right]=\omega^{2}\left[\begin{array}{c}
\frac{i_{0}}{C_{0} \omega_{0}^{2}} \\
\frac{i p_{1}}{C_{P} \omega_{P}^{2}} \\
\frac{i_{1}}{C_{0} \omega_{0}^{2}} \\
\frac{i p_{2}}{C_{P} \omega_{P}^{2}} \\
\frac{i_{2}}{C_{0} \omega_{0}^{2}}
\end{array}\right] .
$$

Eigenvectors are currents scaled with capacitances and element frequencies squared. Eigenvalues are mode frequencies squared.

The voltage through a gap is proportional to the current divided by the capacitance $C_{0}$ of the gap $V_{0, i} \propto I_{0, i} / C_{0, i}$, and the average field $E_{0}$ is defined by voltage divided by cell length $E_{0, i}=V_{0, i} / L$ cell $_{i}$. In the case of all identical cells, currents, voltages, and fields are equivalent, but it is important to consider different definitions in the case of increasing gap lengths or gap perturbations.

This circuit gives a first idea of the PCs stabilization mechanism.

Let us use a transport matrix notation for voltages $V_{n}$ and currents $I_{n}$ along the circuit chain [5]:

$$
\left[\begin{array}{c}
V_{n+1} \\
I_{n+1}
\end{array}\right]=\left[\begin{array}{cc}
1 & -Z_{n} \\
-Y_{n} & 1+Y_{n} Z_{n}
\end{array}\right]\left[\begin{array}{l}
V_{n} \\
I_{n}
\end{array}\right],
$$

where $Z_{n}=\frac{1}{j \omega C_{0}}\left[1-\left(\omega^{2} / \omega_{0}^{2}\right)\right] \quad$ and $\quad Y_{n}=j \omega\{C+$ $\left.C_{p}\left[\omega_{p}^{2} /\left(\omega_{p}^{2}-\omega^{2}\right)\right]\right\}$.

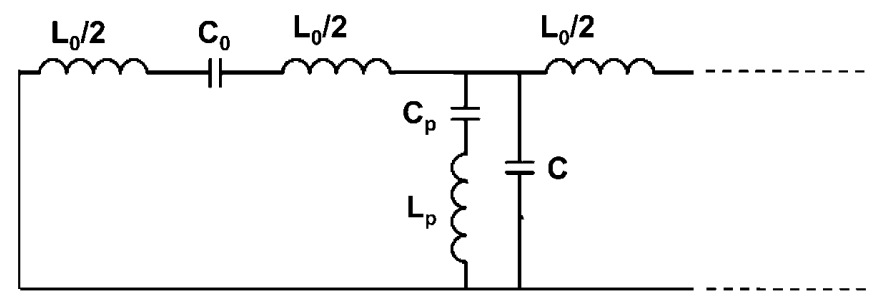

FIG. 15. DTL end plates are represented by short circuit boundary conditions in the equivalent circuit.
The DTL tank is close with metallic end plates. In the equivalent circuit this condition is represented by a short circuit at both ends of the circuit chain (Fig. 15). Then boundary conditions are $V_{0}=0$ and $V_{\text {end }}=0$.

If the first cell capacitance has a perturbation $d C_{0}$, and there are no PCs, the field flatness of the whole structure is perturbed (the quantity proportional to the gap field is the current):

$$
\begin{aligned}
{\left[\begin{array}{l}
V_{1} \\
I_{1}
\end{array}\right]=} & {\left[\begin{array}{c}
-Z_{0} I_{0} \\
\left(1+Z_{0} Y_{0}\right) I_{0}
\end{array}\right]=\left[\begin{array}{c}
-\frac{1}{j \omega C_{0}}\left(d-\frac{\omega^{2}}{\omega_{0}^{2}}\right) I_{0} \\
{\left[1+\frac{j \omega C}{j \omega C_{0}}\left(d-\frac{\omega^{2}}{\omega_{0}^{2}}\right)\right] I_{0}}
\end{array}\right] } \\
= & {\left[\begin{array}{c}
-\frac{1}{j \omega C_{0}}(d-1) I_{0} \\
\left(1+\frac{C}{C_{0}}(d-1)\right) I_{0}
\end{array}\right], }
\end{aligned}
$$

where $d=\frac{C_{0}}{C_{0}+d C_{0}}$ and the driving frequency is $\omega=\omega_{0}$. For the next unperturbed cells the impedance $Z_{n}=0$, and we obtain

$$
\begin{aligned}
I_{2} & =-Y_{1} V_{1}+\left(1+Y_{1} Z_{1}\right) I_{1}=Y_{1} Z_{0} I_{0}+I_{1} \\
& =I_{1}+\frac{C}{C_{0}}(d-1) I_{0} .
\end{aligned}
$$

The difference $\left|I_{n}-I_{n-1}\right|=\left|\frac{C}{C_{0}}(d-1) I_{0}\right|$ is constant along the cell gaps (Fig. 16).

Moreover, if the cell length scales with $\beta \lambda$, the perturbation effect at other cells becomes stronger where the 


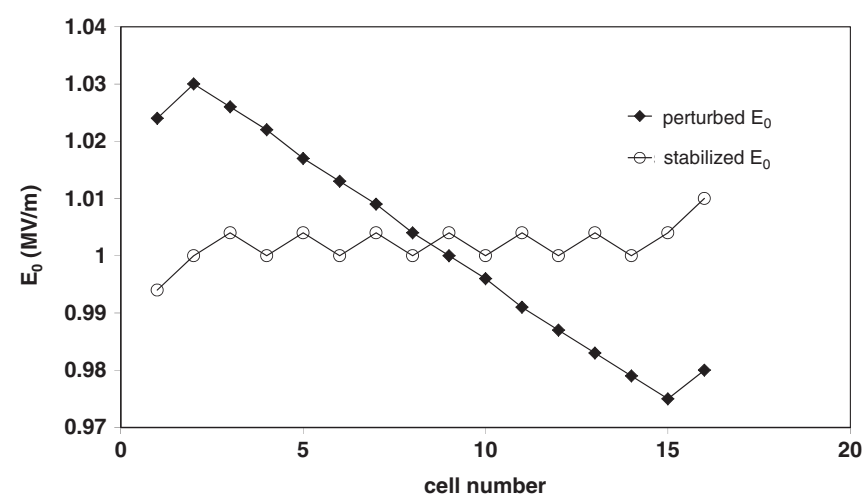

FIG. 16. Perturbed $E_{0}$ without PCs and with PCs at stabilizing value of $C_{p}$ from a circuit simulation. $E_{0}$ is here normalized to 1 , with respect to the nominal value.

ratio $C / C_{0}$ is larger. The effect is therefore stronger towards higher beam energies in the DTL.

Let us now suppose to have one PC every other cell (Fig. 16):

$$
I_{2}=I_{1}+\left[\frac{C}{C_{0}}(d-1)+\frac{C_{p}}{C_{0}}(d-1)\left(\frac{\omega_{p}^{2}}{\omega_{p}^{2}-\omega_{0}^{2}}\right)\right] I_{0} .
$$

The formula shows that: (i) if $d=1$, the presence of the PCs does not affect the field on the gap; (ii) in case of perturbation $(d \neq 1)$ the term introduced by the PCs $\frac{C_{p}}{C_{0}} \times$ $(d-1)\left[\omega_{p}^{2} /\left(\omega_{p}^{2}-\omega_{0}^{2}\right)\right]$ can cancel the term $\frac{C}{C_{0}}(d-1)$ introduced by the perturbation.

The PCs stabilize if, after every PC, the field is set back to the nominal value. Since we suppose to have one PC every other cell, PCs stabilize if $I_{2}=I_{0}$ :

$$
\begin{aligned}
I_{2} \equiv & \left(1+\frac{C}{C_{0}}(d-1)\right) I_{0} \\
& +\left[\frac{C}{C_{0}}(d-1)+\frac{C_{p}}{C_{0}}(d-1)\left(\frac{\omega_{p}^{2}}{\omega_{p}^{2}-\omega_{0}^{2}}\right)\right] I_{0}=I_{0}
\end{aligned}
$$

and from this equation a condition for the optimum value of the PC frequency is obtained:

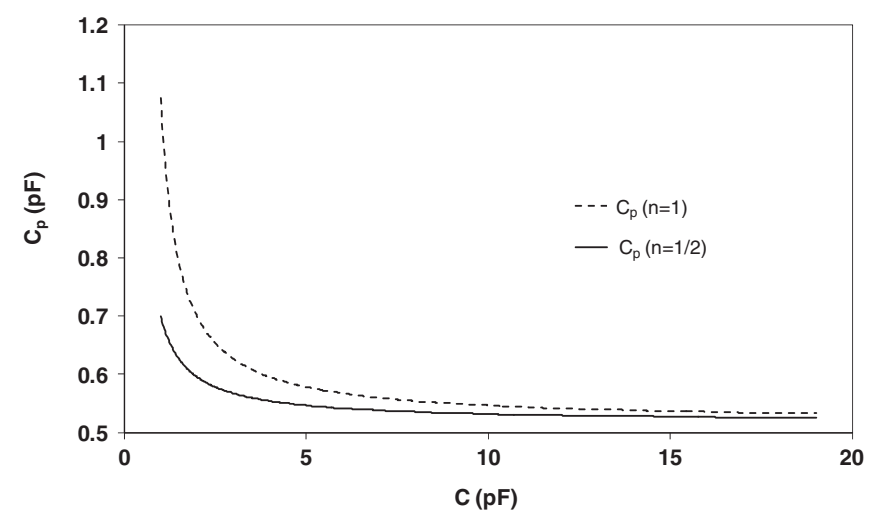

FIG. 17. $\quad C_{p}$ as a function of C (case of $n=1$ and $n=1 / 2$ ).

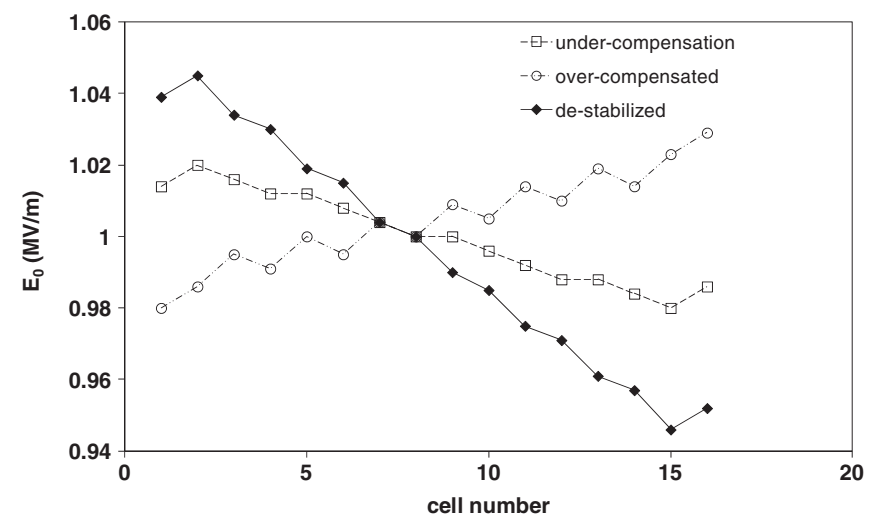

FIG. 18. The PC effect in a perturbed field with gap PC-DT at 3 different configurations described above.

$$
\omega_{p}^{2}=\frac{2 C \omega_{0}^{2}}{2 C+C_{p}} .
$$

If we assume that, when changing the gap PC-DT, the variation of $L_{p}$ is negligible with respect to the
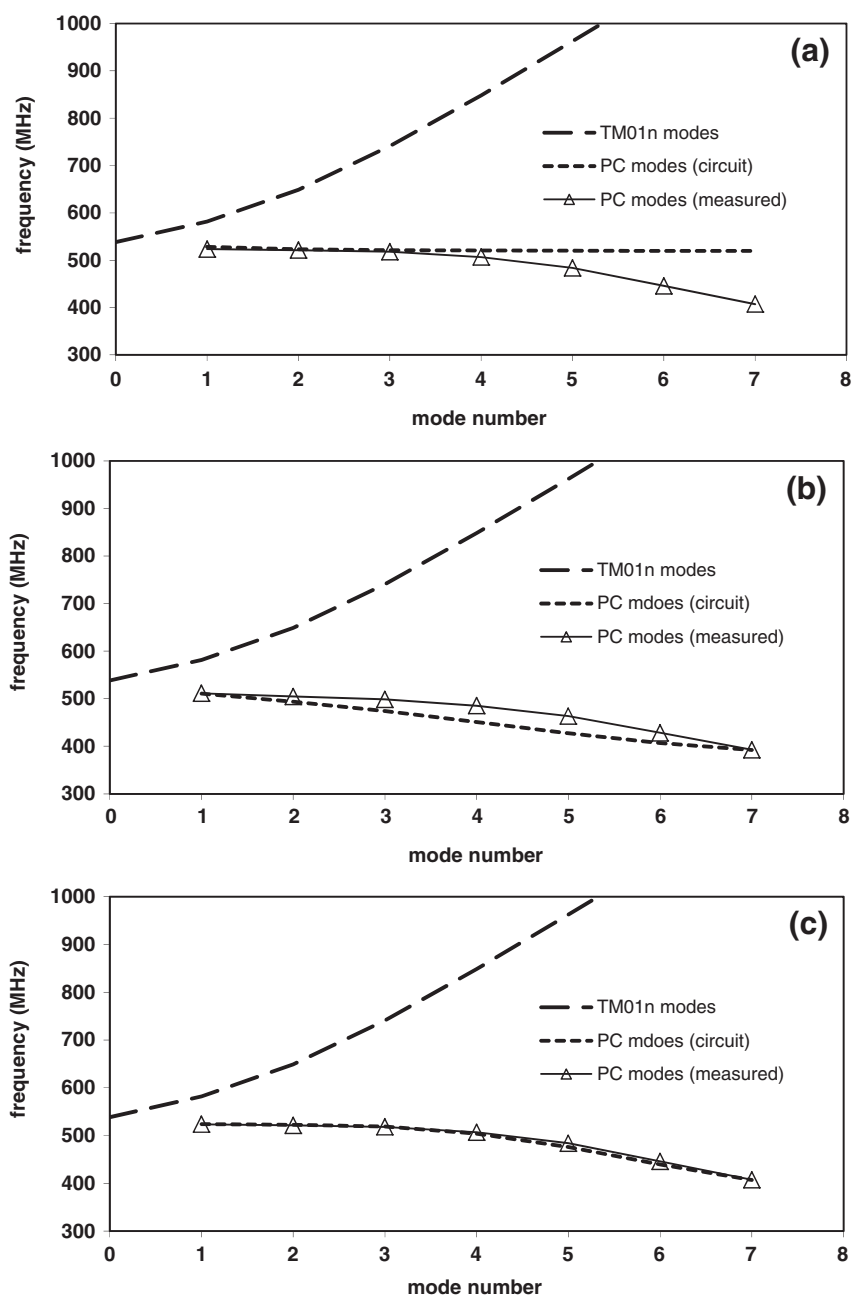

FIG. 19. PC dispersion curve computed by equivalent circuit without PC coupling (a), with nearest neighbor PC coupling (b), and next nearest neighbor PC coupling (c). 


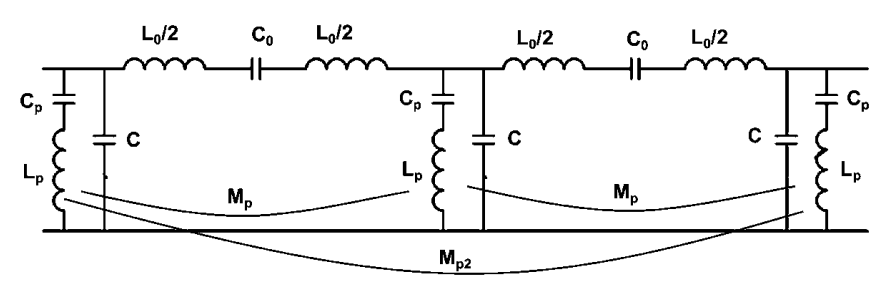

FIG. 20. Equivalent circuit including nearest and next nearest coupling between PCs.

variation of $C_{p}$, it is useful to solve the previous relation for $C_{p}$ :

$$
C_{p}^{\mathrm{stab}}=\frac{2 C}{2 C L_{p} \omega_{0}^{2}-1}
$$

In general, the conditions are $\omega_{p}^{2}=\omega_{0}^{2} /\left(1+\frac{n^{\cdot} C_{p}}{C}\right)$ and $C_{p}^{\text {stab }}=\frac{C}{C L_{p} \omega_{0}^{2}-n}$, where $n=\frac{\text { number postcouplers }}{\text { number cells }}$. Let us define the coupling coefficient $k_{p}=\frac{C_{p}}{C}$ and the previous relation becomes $\omega_{p}^{2}=\omega_{0}^{2} /\left(1+n \cdot k_{p}\right)$, where both $\omega_{p}$ and $k_{p}$ depend on $C_{p}$.

The optimum value of $C_{p}$ for stabilization decreases as a function of the capacitance $C$ (Fig. 17). Where DTs are longer, gap PC-DT must be larger; where DTs are shorter, gap PC-DT must be smaller.

The circuit model reproduces the $E_{0}$ field as a function of different settings of gap PC-DT, as experienced on measurements (Fig. 18): (i) if the gap PC-DT is shorter than the stabilizing condition $\left(C_{p}>C_{p}^{\text {stab }}\right.$ and $\left.\omega_{p}<\omega_{0}\right)$, PCs can compensate the perturbation, but not completely (Fig. 18, square bullets); (ii) if the gap PC-DT is larger than the stabilizing condition $\left(C_{p}<C_{p}^{\text {stab }}\right.$ with $\omega_{0}>\omega_{p}>$ $\left.\omega_{p}^{\text {stab}}\right)$, PCs cause an overcompensation that reverses the slope of the field with respect to the perturbation (Fig. 18, circle bullets); (iii) if gap PC-DT is larger than the stabilizing condition and furthermore the $\mathrm{PC}$ frequency exceeds $\omega_{0}\left(C_{p}<C_{p}^{\text {stab }}\right.$ and $\left.\omega_{p}>\omega_{0}\right)$, the PC effect is destabilizing (Fig. 18, diamond bullets).

A comparison of measured PC dispersion curve with curve obtained by the equivalent circuit [Fig. 19(a)] shows that one needs to take into account a nearest neighbor coupling between PCs, which couples PCs placed in opposite sides of the DTL tank. This nearest neighbor coupling is equivalent to a magnetic coupling between PC inductors $L_{p}$ (Fig. 20) with the coupling factor $k_{p 1}=$ $-\left(M_{p} / L_{p}\right) \cdot \omega_{0}^{2}$ at the operating frequency using self and mutual inductances $L_{p}$ and $M_{p}$.

A next nearest neighbor coupling factor $k_{p 2}$ between PCs is included in the matrix as well. It is equivalent to a mutual inductance $M_{p 2}$ acting between PCs placed at the same side of the DTL tank. $M_{p 2}$ has an opposite sign with respect to $M_{p}$.

Figures 19(a) and 19(b) show the dispersion curves obtained taking into account the PC couplings, Fig. 20 shows the circuit including PC couplings, and Fig. 21 shows the related matrix.

\section{TUNING PROCEDURE AND MEASUREMENTS}

A DTL cold model with scaled dimensions has been built by KASCT in Saudi Arabia for Linac4 (Fig. 22) [6]. The operating frequency is $538.7 \mathrm{MHz}$, the tank diameter $340 \mathrm{~mm}$, the DT diameter $58.8 \mathrm{~mm}$, and the length is

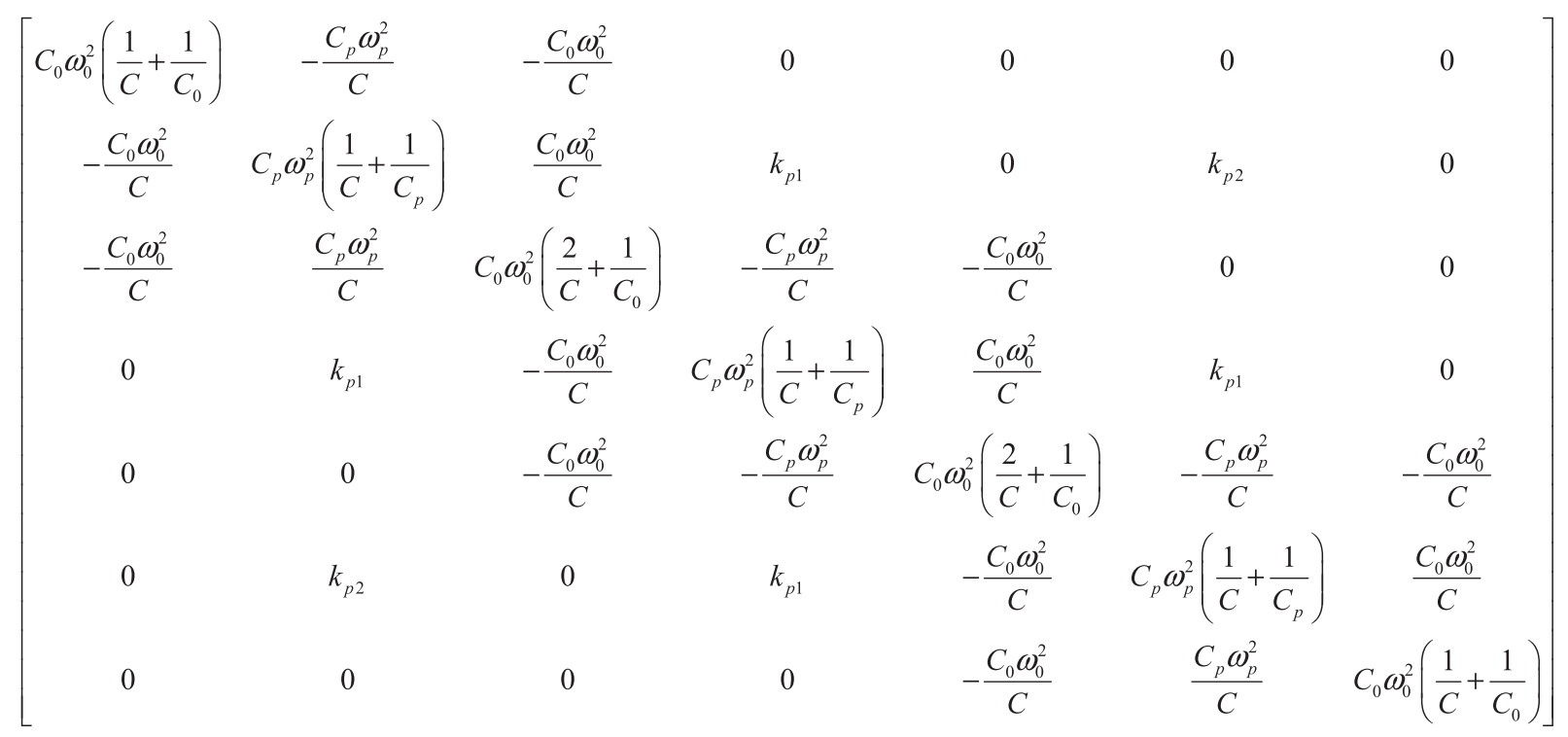

FIG. 21. Circuit matrix including nearest and next nearest coupling between PCs. 

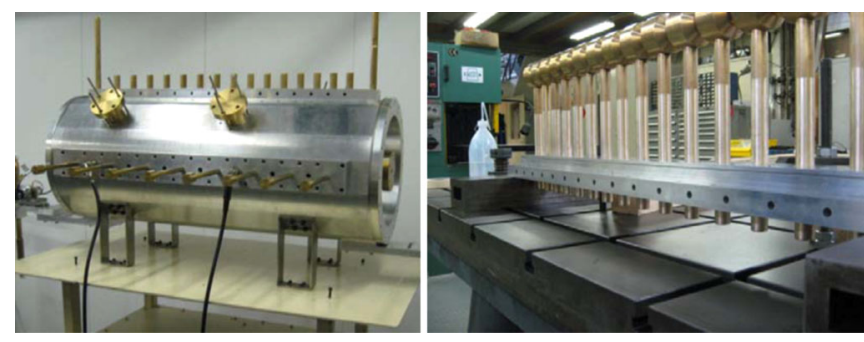

FIG. 22. DTL cold model at CERN and a view of the drift tubes in the alignment phase.

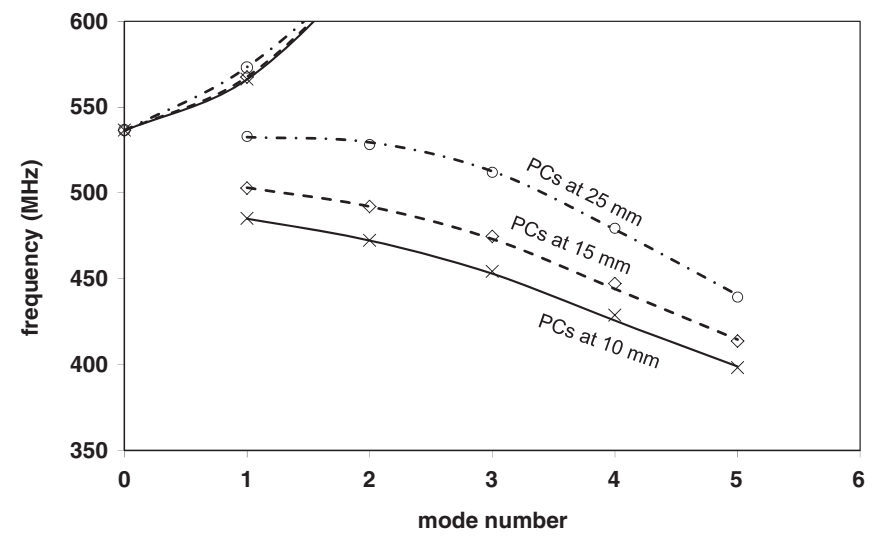

FIG. 23. Frequencies at 3 different values of the gap PC-DT. A larger gap corresponds to higher PC frequencies and to a larger frequency distance between $\mathrm{TM}_{010}$ and $\mathrm{TM}_{011}$ modes.

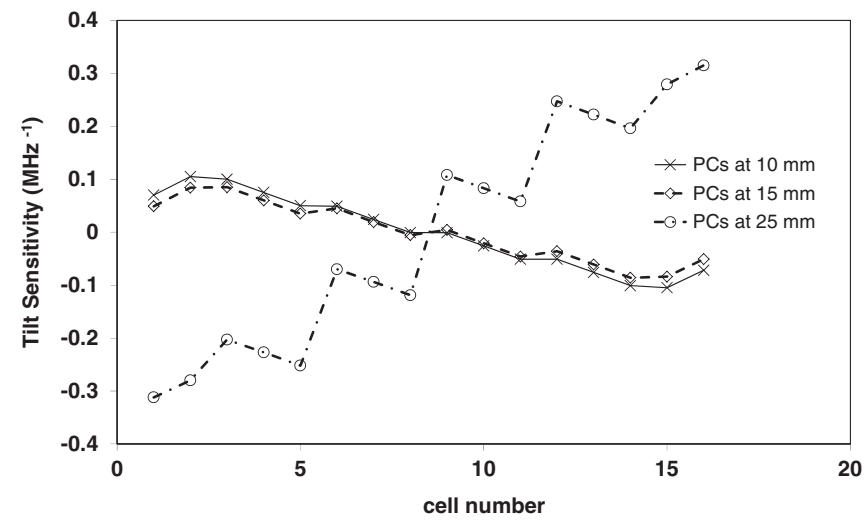

FIG. 24. Tilt sensitivity of perturbed fields as calculated by the equivalent circuit. Each curve is associated to a different value of the gap PC-DT.
$862 \mathrm{~mm}$. It consists of a 16 cells cavity equipped with holes for PC positioning at each DT, in order to test the effectiveness of stabilization for different PC configurations. The end cells can be easily moved in order to induce a perturbation on the accelerating field.

The following results are deduced from the 16 cell DTL cold model present at CERN, equipped with 5 and 7 PCs. Because PCs are every 3 or every 2 drift tubes, we minimize the average value of the tilt sensitivity, resulting in a sawtooth pattern. Tilt sensitivity is defined as $\mathrm{TS}_{i}=$ $\left(E_{0 i}^{\text {pert }}-E_{0 i}^{\text {unpert }}\right) / E_{0 i}^{\text {unpert }} \cdot(1 / \Delta f)$ and tilt sensitivity slope (TS_Slope) is the slope of the linear interpolation of the tilt sensitivity curve.

A procedure to find an optimum average PC length is the following: (i) the gap capacitance $C_{0}$ is calculated from a SUPERFISH [7] simulation, using the formula $\frac{1}{2} C_{0} V^{2}=U$; (ii) measurement of the $\mathrm{TM}_{010}$ and $\mathrm{TM}_{011}$ without PCs to calculate the coupling capacitance $C$ between tank and drift tubes (note: here we make the assumption that all the cells have the same average length, we do not take into account the increasing cell length); (iii) measurement of the $\mathrm{PC}$ frequency band (at least 3 modes, for example $\mathrm{PC}_{\text {highest }}, \mathrm{PC}_{\text {lowest }}, \mathrm{PC}_{\text {central }}$ modes) and of the $\mathrm{TM}_{010}$ and $\mathrm{TM}_{011}$ modes, at different lengths of the PCs (in Fig. 23, three different lengths have been considered); (iv) fitting of the measured frequencies with dispersion curves computed by the circuit, in order to obtain the circuit parameters $\omega_{0}, \omega_{p}, C_{p}, k_{p 1}$, $k_{p 2}$ (now we have a complete set of circuit parameters for different PC lengths used in measurements); (v) insertion of a perturbation $\delta C_{0}$ in the end capacitances of the circuit, which simulates the end cell displacement; (vi) for every PC length, the circuit simulates $E_{0}$ field and tilt sensitivity in the presence of the perturbation $\delta C_{0}$ (Fig. 24); (vii) if none of the considered PC lengths makes the tilt sensitivity flat, the stabilizing PC length can be extrapolated from the parameter curves (Table I), looking at which PC length the TS_Slope curve crosses the zero (Fig. 25).

The same analysis has been done for both 5 PCs configuration and 7 PCs configuration.

In order to verify the equivalent circuit results, we use the tilt sensitivity method to find the stabilization length on the DTL cold model equipped with 5 and 7 PCs.

TABLE I. Stabilizing PC setting for DTL cold model equipped with 5 PCs and 7 PCs.

\begin{tabular}{lccccccr}
\hline \hline & & \multicolumn{3}{c}{ Stabilizing parameters } & & & \\
& Gap PC-DT $[\mathrm{mm}]$ & $\omega_{p}[\mathrm{MHz}]$ & $C_{p}[\mathrm{pF}]$ & $k_{0} / \omega_{0}{ }^{2}$ & $k_{p} / \omega_{0}{ }^{2}$ & $k_{p 1} / \omega_{0}{ }^{2}$ & $k_{p 2} / \omega_{0}{ }^{2}$ \\
\hline 5 postcouplers & 22.5 & 485 & 0.62 & 2.3 & 0.20 & 0.087 & -0.033 \\
7 postcouplers & 19.0 & 477 & 0.61 & 2.3 & 0.19 & 0.096 & -0.044 \\
\hline \hline
\end{tabular}



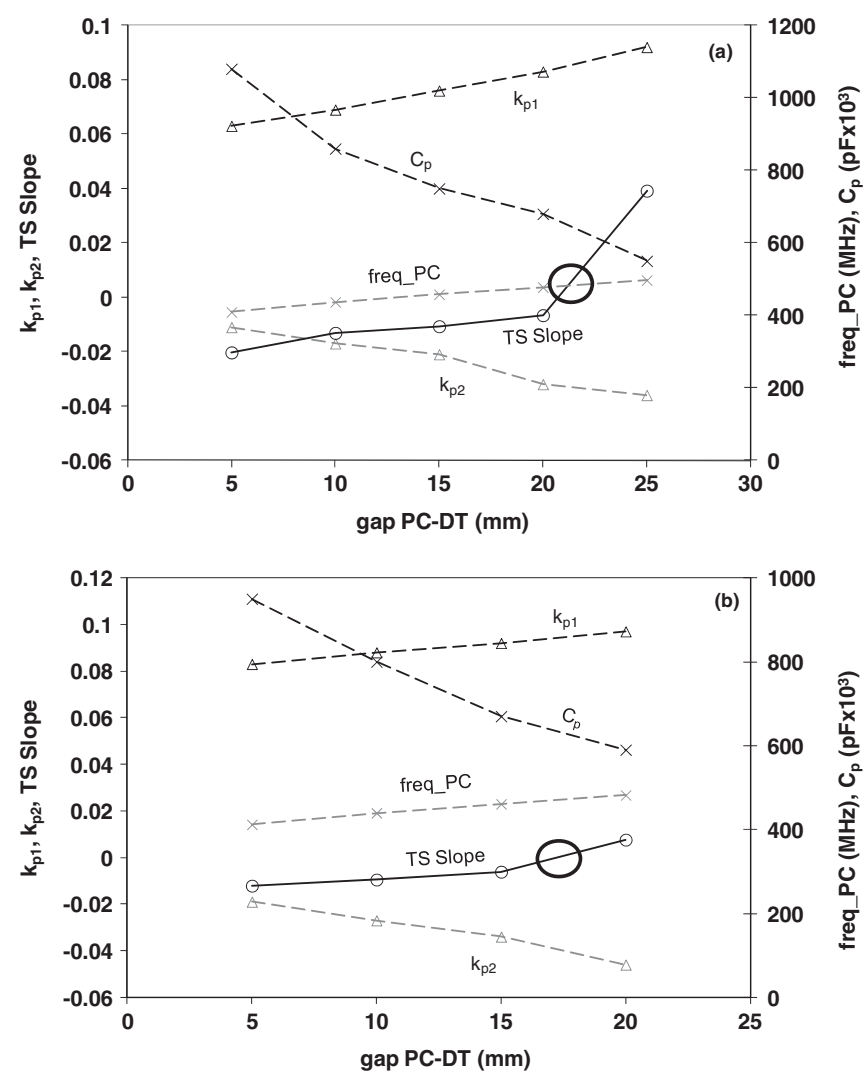

FIG. 25. Circuit parameters and tilt sensitivity slope as a function of the gap PC-DT for 5 PC (a) and 7 PC (b) configurations. The circles mark the value of gap PC-DT where the TS_Slope crosses the zero point. It should be the stabilizing gap PC-DT.

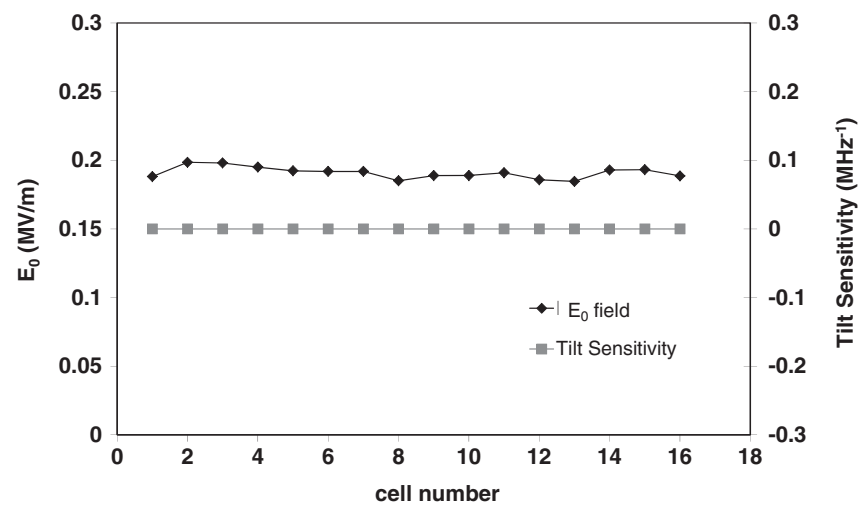

FIG. 26. Reference field and tilt sensitivity.

First, we need a measurement of the reference field (Fig. 26) to be stabilized, which is called the "natural field distribution" of the tank. In order to verify the effectiveness of PCs for stabilization of the accelerating field, it is not necessary to refer to a perfectly flat field.

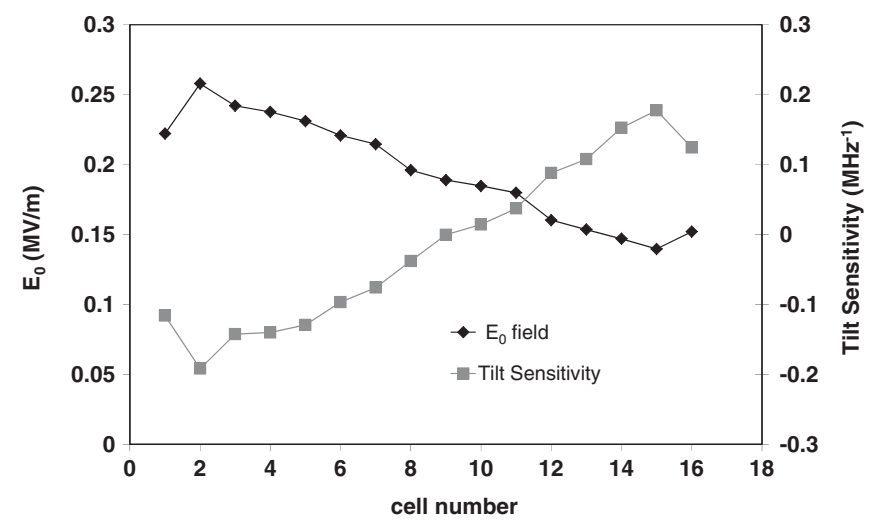

FIG. 27. Perturbed field and tilt sensitivity.
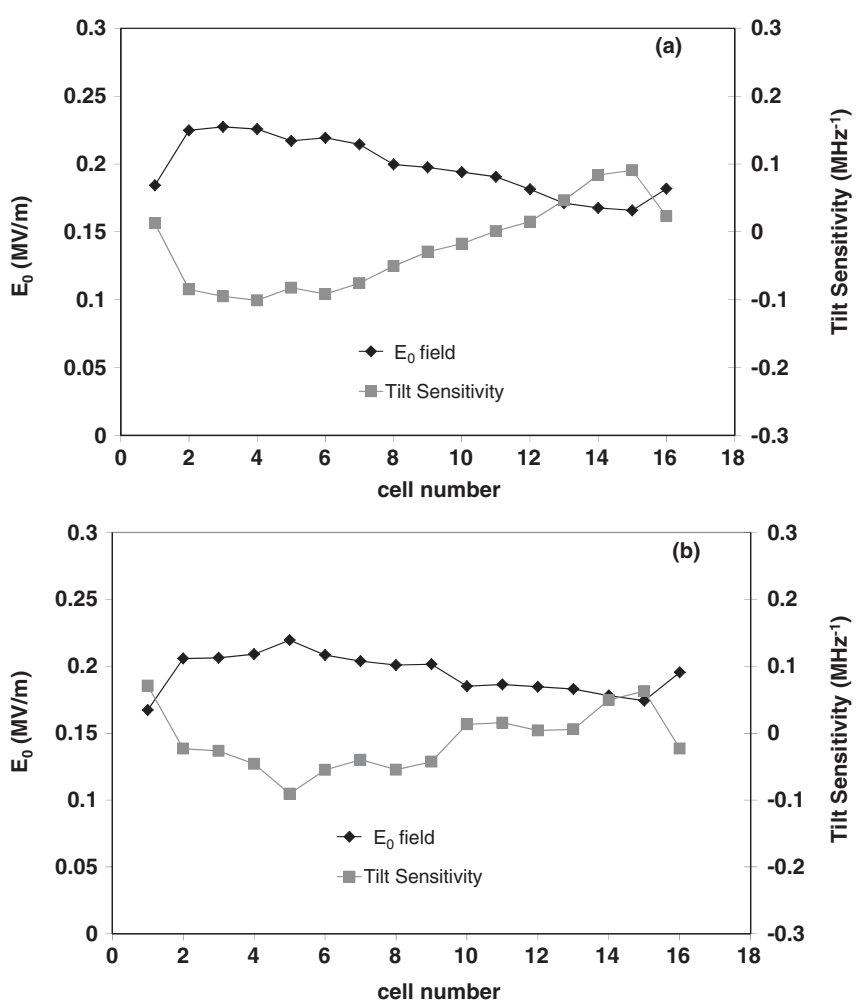

FIG. 28. Gap PC-DT $=15 \mathrm{~mm}$ is too small for both 5 PCs (a) and 7 PCs (b) case.

We perturb the first and the last gap to create a tilt in the field (Fig. 27).

Now we start with a small gap PC-DT $(15 \mathrm{~mm})$, and as expected from the equivalent circuit, $\mathrm{PCs}$ are not able to stabilize the tilt of the field (Fig. 28).

If gap is too large ( $25 \mathrm{~mm}$ for $5 \mathrm{PCs}, 22 \mathrm{~mm}$ for $7 \mathrm{PCs}$ ), the field reverses its slope, and we reach overcompensation (Fig. 29).

Finally, we set the PCs at gap PC-DT calculated with the equivalent circuit: $22.5 \mathrm{~mm}$ gap for $5 \mathrm{PCs}, 19 \mathrm{~mm}$ gap for 

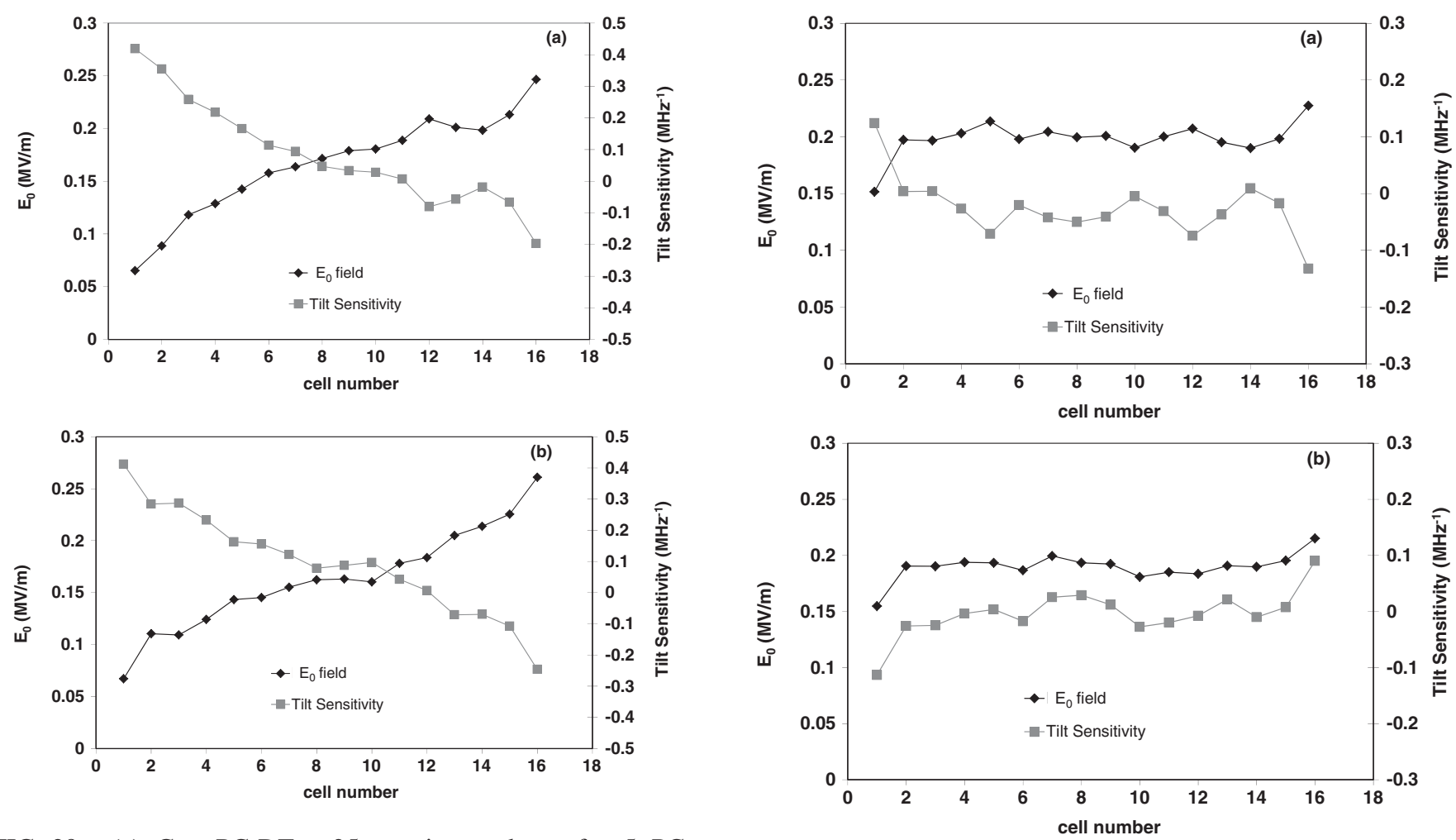

FIG. 29. (a) Gap PC-DT $=25 \mathrm{~mm}$ is too large for $5 \mathrm{PCs}$ configuration. (b) Gap PC-DT $=22 \mathrm{~mm}$ is too large for 7 PCs configuration.
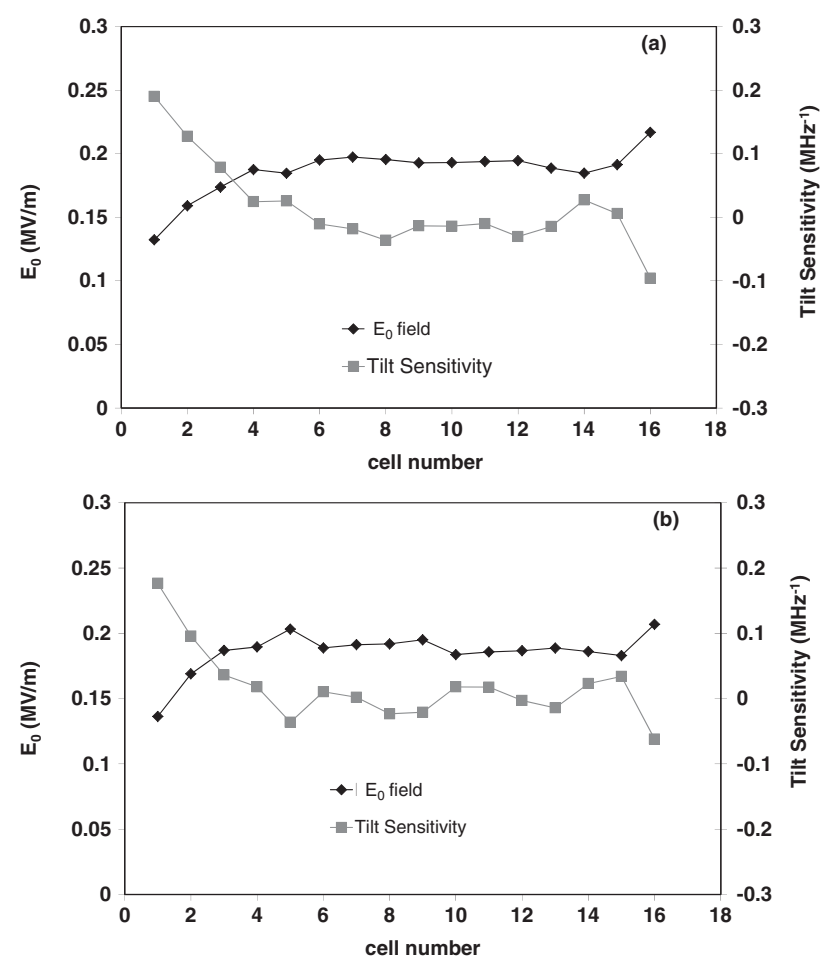

FIG. 30. (a) 5 PCs are set all at the $\operatorname{gap}_{\mathrm{pc}}=22.5 \mathrm{~mm}$, as calculated by the equivalent circuit simulation (Fig. 27). (b) 7 PCs are set all at the gap $_{\mathrm{pc}}=19 \mathrm{~mm}$, as calculated by the equivalent circuit simulation (Fig. 27).

FIG. 31. PCs are adjusted, taking into account the increasing length of the cells for 5 PCs (a) and 7 PCs (b).

7 PCs. One can see the tilt sensitivity curve approaching to zero, except that for the cells close to the tank ends (Fig. 30).

This effect can be mitigated by adjusting the gap PC-DT in order to take into account the increasing cell length in this model. What we expect is an optimum $\mathrm{PC}$ configuration where the central $\mathrm{PC}$ is kept at the computed gap PC-DT, but with smaller gap PC-DT at the low energy side and larger gap PC-DT at the high energy side (Fig. 31). Table II shows the final PC settings.

Let us now digress on the $\mathrm{PC}$ length tuning, considering the ratio $r=\frac{\Delta\left(\mathrm{TS}_{\text {Slope }}\right)}{\Delta \text { gap }_{\mathrm{PC}}-\mathrm{DT}}$ as a parameter for the PC length adjustment difficulty. This parameter is larger in the case of 5 PCs, where $r=9$ around the optimum gap $=22.5 \mathrm{~mm}$, than in the case of 7 PCs, where $r=2$ around the optimum gap $=19 \mathrm{~mm}$. In the case of a larger gap PC-DT, a larger

TABLE II. Optimum PC settings for DTL cold model at CERN.

\begin{tabular}{lccccccc}
\hline \hline PC number & 1 & 2 & 3 & 4 & 5 & & \\
Gap [mm] & 21.5 & 22 & 22.5 & 23 & 23.5 & & \\
PC number & 1 & 2 & 3 & 4 & 5 & 6 & 7 \\
Gap [mm] & 17.5 & 18 & 18.5 & 19 & 19.5 & 20 & 20.5 \\
\hline \hline
\end{tabular}


precision is required to fix the optimum PC length for stabilization.

\section{CONCLUSIONS}

The equivalent circuit for a DTL equipped with PCs is exhaustive: it includes all the elements of the cavity, it gives an explanation of the stabilizing PC mechanism in terms of circuit parameters and shows that PC frequency tuning is indissolubly related to the PC-DT coupling with the relation between $C_{p}$ and $\omega_{p}$.

The matrix form of circuit equations allows the introduction of nearest neighbor coupling and next nearest neighbor coupling between PCs. With the insertion of couplings, experimental dispersion curves are precisely reproduced.

3D simulations were undertaken to study PCs mode frequencies as a function of number of PCs per meter and of the gap PC-DT. In quasistatic approximation, formulas for estimation of PC circuit parameters as a function of the PC geometry were defined and verified to be consistent with simulation results.

A tuning procedure based on the equivalent circuit and on frequency measurements was defined. The advantage of this method with respect to the standard method [8] is in avoiding a large number of bead pulling measurements at different PC lengths. The predictions of the circuit are verified with measurements on the DTL cold model, in cavity configurations with 5 and 7 PCs.

\section{ACKNOWLEDGMENTS}

This work has been mostly done at CERN, in the framework of the Linac4 project. I would like to thank G. De Michele, S. Ramberger, and M. Vretenar for their support.

[1] D. Swenson et al., in Proceedings of High-energy Accelerators Conference, Cambridge, MA, 1967.

[2] Ansoft Corporation, HFSS 10.1.

[3] T. Wangler, RF Linear Accelerators (John Wiley \& Sons, Inc., New York, 1998), p. 5.14.

[4] F. Grespan et al., in Proceedings of the 23rd Particle Accelerator Conference, Vancouver, Canada, 2009 (IEEE, Piscataway, NJ, 2009).

[5] T. Wangler, RF Linear Accelerators (John Wiley \& Sons, Inc., New York, 1998), p. 3.4.

[6] N. Alharbi, F. Gerigk, and M. Vretenar, Technical Report No. CARE-Note-2006-012-HIPPI, CARE, 2006.

[7] Los Alamos Accelerator Code Group, Poisson Superfish.

[8] J.M. Potter, in Handbook of Accelerator Physics and Engineering, edited by $\mathrm{A}$. Wu Chao and $\mathrm{M}$. Tigner (World Scientific, Singapore, 2006). 